\title{
¿Las plantas exóticas leñosas dependen de los polinizadores para reproducirse? Un estudio de caso en las Sierras de la Ventana (Argentina)
}

\author{
Lucía C. Martínez ${ }^{1,2}$; ; Joana P. Haedo $^{1 / 3} \&$ Hugo J. Marrero ${ }^{1,3}$ \\ ${ }^{1}$ Laboratorio de Interacciones Bióticas en Agroecosistemas (LIBA). ${ }^{2}$ Departamento de Biología, Bioquímica y Farmacia, \\ Universidad Nacional del Sur. Bahía Blanca, Argentina. ${ }^{3}$ Centro de Recursos Naturales Renovables de la Zona Semiárida,
} CONICET. Bahía Blanca, Argentina.

Resumen. En diferentes medidas, la mayoría de las plantas con flores dependen de los polinizadores para su reproducción. En el caso de las especies exóticas, el grado de dependencia es clave, ya que puede afectar su éxito en la colonización de nuevos ambientes. El objetivo de este estudio fue determinar el grado de dependencia de los polinizadores de cinco especies de plantas entomófilas exóticas en pastizales pampeanos de las Sierras de la Ventana. Se determinó si las diferencias en la dependencia se relacionan con la ubicación geográfica y con la filogenia de las especies vegetales. Las especies estudiadas fueron Spartium junceum, Genista monspessulana, Prunus mahaleb, Rosa rubiginosa y Rubus ulmifolius. El muestreo se realizó en el Parque Provincial Ernesto Tornquist (Provincia de Buenos Aires) y en zonas aledañas a éste. En los picos de floración de cada especie y en diferentes sitios se seleccionaron individuos a los que se les realizaron dos tratamientos en flores previamente embolsadas: autopolinización y polinización cruzada. Además, se dejaron flores sin polinizar como controles. Luego, se calculó la probabilidad de formación de frutos y la cantidad de semillas tanto en el control como bajo los diferentes tratamientos. Finalmente, se compararon los resultados de este estudio con otros realizados en diferentes regiones (excepto para $R$. ulmifolius). Las especies de plantas estudiadas mostraron una respuesta variada en cuanto a la dependencia de polinizadores, desde especies muy dependientes (e.g., S. junceum, G. monspessulana y P. mahaleb) hasta otras menos dependientes (e.g., R. rubiginosa y R. ulmifolius). Esto indica que la dependencia de los polinizadores puede ser variable en plantas que estén colonizando un hábitat fuera de su distribución nativa. Adicionalmente, existen variaciones en el grado de dependencia según el área geográfica, excepto para $R$. rubiginosa, lo que muestra que la localización geográfica puede influir sobre la dependencia de polinizadores de las plantas leñosas.

[Palabras clave: Spartium junceum, Genista monspessulana, Prunus mahaleb, Rosa rubiginosa, Rubus ulmifolius, autopolinización, polinización cruzada, invasiones biológicas]

\begin{abstract}
Do exotic plants depend on pollinators for reproduction? A case study in Sierras de la Ventana (Argentina). Most of the flowering plants depend on pollinators, in different degrees, for their reproduction. The degree of pollinator dependence is especially important in exotic plants because it could determine their colonization success in new locations. The objective of this study was to determine the degree of pollinator dependence in five exotic entomophilous plant species in a Pampean grassland located in Sierra de Ventana. It was determined if the differences in dependency are related to the geographic location and the phylogeny of plant species. The plants species were Spartium junceum, Genista monspessulana, Prunus mahaleb, Rosa rubiginosa and Rubus ulmifolius. Sampling was carried out in the Ernesto Tornquist Provincial Park and in surrounding areas (Buenos Aires Province). During the flowering peak of each species and in different sites, two treatments were undertaken in pre-bagged flowers: self-pollination and cross-pollination. Also, others pre-bagged flowers were left without pollination as control. Later, the probability of fruit develop and seed set under the two treatments and the control were assessed. Finally, the results of this study were compared with studies carried out in other regions (except for R. ulmifolius). The studied plant species showed a varied response to pollinator dependence, from very dependent species, such as S. junceum, G. monspessulana and P. mahaleb, to low dependent species such as $R$. rubiginosa y R. ulmifolius. Our results indicate that pollinator dependence can be variable in plants species that are colonizing non-native habitats. Furthermore, there are variations in the degree of pollinator dependency according to the geographical area, except for $R$. rubiginosa, showing that the geographical location can influence the dependence on pollinators for woody plants.
\end{abstract}

[Keywords: Spartium junceum, Genista monspessulana, Prunus mahaleb, Rosa rubiginosa, Rubus ulmifolius, selfpollination, cross-pollination, biological invasions]

Editor asociado: Lucas Garibaldi \ruciamdell@gmail.com
Recibido: 16 de Mayo de 2019

Aceptado: 19 de Septiembre de 2020 


\section{INTRODUCCIÓN}

Los sistemas reproductivos de las plantas varían de forma considerable entre la reproducción sexual y asexual, y abarcan desde especies altamente autoincompatibles hasta especies completamente autógamas (Barrett and Eckert 1990). Los sistemas reproductivos pueden verse modificados dentro de las mismas especies y poblaciones, bajo diferentes factores ecológicos y demográficos (Barrett 2010; Lozada-Gobilard et al. 2019). Los polinizadores son una de las posibles causas de modificación de los sistemas reproductivos (Eckert et al. 2010). Poblaciones de plantas que presentan diferentes tipos de polinizadores (e.g., abejas o moscas) pueden modificar su sistema reproductivo y volverse más o menos dependientes del transporte de polen por los polinizadores (Devaux et al. 2014). Es por eso que la región geográfica podría jugar un rol fundamental en determinar las estrategias reproductivas de una especie vegetal, ya que las interacciones con los polinizadores disponibles en esa región podrían determinar la selección de un fenotipo que resulte más exitoso bajo ese contexto (Gervasi and Schiestl 2017).

Un caso particular de modificación de los sistemas reproductivos puede ocurrir cuando una especie exótica ingresa a una nueva comunidad (Richardson et al. 2000). Existen diversas hipótesis acerca de la relación entre la capacidad de invasión y los sistemas reproductivos de las plantas exóticas. Una de ellas, la Ley de Baker, propone que las plantas autocompatibles, sobre todo las que se autopolinizan sin mediación de polinizadores, están menos limitadas para su establecimiento en un entorno nuevo (Baker 1955; Barrett et al. 1989; Parker and Haubensak 2002). Por lo tanto, el éxito reproductivo de las plantas exóticas podría depender de su capacidad para producir descendencia de forma autógama sin necesidad de polinización cruzada. De lo contrario, las plantas que necesitan ser polinizadas por insectos podrían verse limitadas por la falta de ellos o por la competencia con plantas nativas (Richardson et al. 2000).

Si bien las plantas autocompatibles serían más exitosas al no tener una limitación por polinizadores (Richardson et al. 2000), muchas especies de plantas exóticas (en especial leñosas) dependen mucho de los polinizadores para reproducirse (Memmott and Waser 2002; Traveset and Richardson 2014). El grado en el que estas especies están limitadas por el transporte de polen exógamo mediante vectores bióticos (i.e., cuánto dependen de ellos para su reproducción) es clave, ya que éstos pueden ser limitantes durante la colonización de un nuevo ecosistema (Traveset and Richardson 2014). Por otro lado, si la polinización biótica es una barrera que puede limitar la invasión de las plantas leñosas (Parker 1997; Barret 2010; Ward et al. 2012), el grado de dependencia de los polinizadores debería ser similar en la mayoría de las especies. No obstante, la dependencia de polinizadores podría no ser una generalidad para todas las especies, pero sí verse mejor representada en grupos de especies de plantas filogenéticamente relacionadas (Niet and Johnson 2012).

Conocer los sistemas reproductivos de las plantas exóticas bajo diferentes contextos ecológicos puede resultar importante, ya que las estrategias reproductivas se pueden ver modificadas según la región geográfica donde se desarrollen (Parker and Haubensak 2002). Por ejemplo, Parker y Haubensak (2002) mostraron que la limitación de polen en dos especies invasoras (Cytisus scoparius y Genista monspessulana) variaba según el lugar geográfico y el ensamble de polinizadores con los que interactuaron. De esta forma, una especie muy dependiente de polinizadores en su rango nativo podría modificar la intensidad de la dependencia, o dejar de serlo, durante la colonización en un nuevo ambiente y viceversa (Gervasi and Schiestl 2017). Por este motivo, evaluamos la dependencia de polinizadores de cinco especies de plantas introducidas en pastizales naturales de la Argentina y comparamos nuestros resultados con estudios realizados en otras regiones geográficas donde también se comportan como invasoras. Esta información resultará útil para ampliar el conocimiento sobre las respuestas de los sistemas reproductivos de las plantas (en particular, leñosas) frente a diferentes contextos de invasiones biológicas (Ward et al. 2012).

El objetivo de este estudio fue determinar en qué medida cinco especies de plantas leñosas entomófilas exóticas dependen del transporte de polen exógamo mediado por polinizadores en las Sierras de la Ventana (Buenos Aires, Argentina). Además, se estudió si los cambios en la dependencia de polinizadores se relacionan con la ubicación geográfica y con la cercanía filogenética de estas especies. Nuestra hipótesis es que el grado de dependencia de 
los polinizadores es afectado por la ubicación geográfica de las especies y por su relación filogenética. Esperamos encontrar que (a) las especies dependan en diferente medida de polinizadores; (b) existan diferencias en la dependencia relacionadas a la localización de las poblaciones de las especies, y (c) ocurra una similitud en la dependencia en especies filogenéticamente relacionadas.

\section{Materiales y Métodos}

\section{Área de estudio}

El trabajo se realizó dentro del Parque Provincial Ernesto Tornquist (PPET), ubicado al sudoeste de la provincia de Buenos Aires $\left(38^{\circ} 03^{\prime} 00^{\prime \prime} \mathrm{S}\right.$ - 62 $\left.62^{\circ} 02^{\prime} 00^{\prime \prime} \mathrm{O}\right)$ y en zonas aledañas a éste $\left(38^{\circ} 5^{\prime} 32.93^{\prime \prime} \mathrm{S}-62^{\circ} 3^{\prime} 16.7^{\prime} \mathrm{O}\right.$; 38 7'6.96" S 61 58'4.87' O). El PPET cubre una extensión de 6700 ha, forma parte del Sistema de Ventania y representa uno de los escasos relictos de pastizal pampeano protegido dentro de la provincia (Bilenca and Miñarro 2004). El clima es templado, con una temperatura media anual de $14.6^{\circ} \mathrm{C}$ (Frangi and Bottino 1995). La precipitación anual es $800 \mathrm{~mm}$, concentrada en la primavera (Burgos 1971). Los tipos de vegetación dominante son estepas graminosas (Frangi and Bottino 1995) y comunidades edáficas de gramíneas y dicotiledóneas, con numerosos endemismos. Las familias más representadas son Poaceae y Asteraceae (Long and Grassini 1997). Actualmente, dicha reserva de pastizal pampeano sufre un avance notorio por parte de especies de plantas leñosas exóticas, que lograron establecerse y aumentan sus densidades poblacionales año a año (Zalba and Villamil 2002; Sanhueza 2012; Ramírez 2020). El avance de especies exóticas constituye la principal amenaza para la conservación de la diversidad de flora y fauna nativa dentro del área protegida (Zalba and Villamil 2002).

\section{Especies de plantas estudiadas}

La elección de las especies estuvo determinada en base a dos criterios. El primero fue la disponibilidad de poblaciones dentro del PPET y en las inmediaciones, y el segundo fue el grado de avance de estas especies dentro de la reserva en los últimos años (Zalba and Villamil 2002; Sanhueza 2012; Amodeo and Zalba 2013; Ramírez 2020).

Spartium junceum (retama) y Genista monspessulana (retamilla) son especies de arbustos perennes que pertenecen a la familia Fabaceae, nativas de la región del Mediterráneo y de las islas Canarias y Azores (Allen and Allen 1981; Clapham et al. 1987; Pauchard et al. 2008). Su reproducción es exclusivamente sexual. Ambas especies necesitan ser visitadas por insectos para que empujen la quilla y se liberen los estambres (Parodi 1978; Parker and Haubensak 2002). Durante el desarrollo de este trabajo se observaron diferentes especies de abejas (Apis mellifera, Xylocopa sp. y Bombus sp.) visitando las flores de ambas especies.

Prunus mahaleb (cerezo de Santa Lucía) es un árbol de mediano porte perteneciente a la familia Rosaceae.Su distribución nativaincluye el centro y el sur de Europa, el noroeste de África y el oeste de Asia central (Jordano 1994). Su reproducción es exclusivamente sexual y su dispersión es sobre todo por zoocoria. Estudios recientes indican la presencia de individuos con flores androestériles (ca. 5\%) en las poblaciones del PPET, aunque la mayor proporción son hermafroditas (Amodeo and Zalba 2017). Se observaron diversos visitantes florales, himenópteros tales como Apis mellifera, Bombus sp. y Polybia scutellaris, dípteros como Allograpta exotica, Copestylum sp. y Oxysarcodexia varia, un coleóptero Astylus quadrilineatus y un lepidóptero Vanessa braziliensis.

Rosa rubiginosa (rosa mosqueta) es una especie arbustiva decidua que pertenece a la familia Rosaceae. Es nativa de Europa y de Asia (Mazzolari et al. 2016). Numerosos estudios demostraron que puede reproducirse de forma asexual mediante apomixis (Mazzolari et al. 2016). Durante el desarrollo del trabajo se observaron visitantes florales en esta especie, en particular Apis melífera y Astylus quadrilineatus.

Rubus ulmifolius (zarzamora) es un arbusto espinoso y voluble también perteneciente a la familia Rosaceae, nativo de Europa y del norte de África (Monasterio-Huelin and Weber 1996). Puede reproducirse de forma vegetativa a partir de porciones del tallo, por lo general luego de algún disturbio a nivel del individuo. Su reproducción es muy eficiente, ya que cada flor es capaz de producir muchas semillas y sus frutos son consumidos por aves y pequeños mamíferos que ayudan en la dispersión (Mazzolari and Comparatore 2014). En el área de estudio se observó a esta especie con abundantes visitantes florales, entre ellos Apis mellifera, Xylocopa sp., Allograpta exotica, Astylus quadrilineatus y Polybia scutellaris. 


\section{Diseño de muestreo}

Para cada una de las especies de plantas se seleccionaron nueve sitios separados por una distancia mínima de 200 m, excepto para rosa mosqueta, para la cual solo se seleccionaron seis sitios. En cada sitio se eligieron al azar entre 2 y 4 individuos por especie, a los que se les realizaron ensayos de polinización para evaluar la dependencia de los polinizadores. En total se muestrearon 5 flores por individuo en cada tratamiento, siempre que fue posible (ver ensayos de polinización). El número de flores total para cada especie fue variable debido a diferentes factores ambientales. Para el caso de rosa mosqueta, dada la menor cantidad de pimpollos florales encontrados, la cantidad de flores muestreadas por individuo fue entre 2 y 3 para cada tratamiento. Los ensayos de polinización se realizaron entre septiembre y diciembre del año 2017 para $S$. junceum, $P$. mahaleb y $R$. rubiginosa, y entre septiembre y diciembre del año 2018 para G. monspessulana y R. ulmifolius. Los frutos fueron recolectados una vez que se formaron completamente, pero antes de la dehiscencia en el caso de las leguminosas.

\section{Ensayos de polinización}

Para estimar la dependencia de polinizadores utilizamos la metodología propuesta por Kearns e Inouye (1993). En cada individuo se colocaron bolsas de tul en ramas que contenían sólo botones florales. De esta manera se evitó la visita de cualquier insecto una vez que las flores estuvieran receptivas. Colocar las bolsas de tul no evitó que llegue polen exógamo mediante otros vectores como el viento o el agua, pero aseguró que las diferencias encontradas en los tratamientos se deban a la deposición de polen a través de un vector biótico. Una vez que las flores o inflorescencias (en adelante flores) embolsadas se encontraron maduras (7-10 días luego del embolsado), a cada una de ellas se le realizó uno de los siguientes tratamientos: polinización cruzada (PC; deposición de polen perteneciente a una flor de otro individuo sobre el estigma de la flor tratada) o autopolinización (A; deposición de polen perteneciente a la misma flor tratada sobre su estigma). Además, se dejaron flores sin manipular como control (C). Los tratamientos y el control se realizaron en una misma bolsa cuando fue posible, de manera que la misma bolsa contuvo aproximadamente la misma cantidad de flores tratadas y control. Se tuvo precaución para evitar el riesgo de polinización entre flores de distintos tratamientos dentro de las bolsas. Todas las flores fueron etiquetadas para su posterior identificación.

Una vez realizados los tratamientos, se colocaron nuevamente las bolsas de tul para evitar las visitas de polinizadores a las flores manipuladas. No se trabajó con flores sin embolsar para evaluar el servicio de polinización, ya que éste puede modificarse por variaciones climáticas estacionales y podría no relacionarse a la dependencia de los polinizadores de las plantas (Petanidou et al. 2014). Entonces, el servicio de polinización podría ser bueno o malo en años diferentes para un individuo de una especie de planta, pero la dependencia de los polinizadores en ese individuo debería ser la misma. La cantidad total de flores manipuladas varío dependiendo de la especie y el tratamiento (ver Tabla S1 en los Materiales Suplementarios). El bajo número de tratamientos en rosa mosqueta se debió a la pérdida de datos por problemas metodológicos.

Una vez formados los frutos o infrutescencias (en adelante frutos), se recolectaron y se transportaron al laboratorio, donde se cuantificó la cantidad de frutos y semillas formados bajo cada tratamiento y para cada una de las especies. En R. rubiginosa y R. ulmifolius se consideró la cantidad de infrutescencias y la cantidad de frutos por infrutescencia, como el equivalente a la cantidad de frutos y de semillas por fruto.

\section{Análisis de datos}

Para estimar la dependencia de polinizadores de cada una de las especies bajo los diferentes tratamientos, se analizaron los datos a partir de modelos lineales generalizados y mixtos (GLMM por sus siglas en inglés). Se realizaron dos modelos por especie, uno donde la variable respuesta fue la probabilidad de que se forme un fruto bajo los distintos tratamientos y otro donde la variable respuesta fue el número de semillas formadas por fruto bajo los distintos tratamientos. Para la variable de probabilidad de formación de frutos se tuvo en cuenta cada flor en particular, con una probabilidad de uno para frutos formados y cero para frutos no formados. Los modelos fueron generados utilizando el comando glmer del paquete lme4 del software estadístico R (Core Team 2013), con distribución binomial para la variable frutos y distribución Poisson para la variable semillas. Tres especies (G. monspessulana, $R$. rubiginosa y $R$. ulmifolius) mostraron gran sobredispersión en los datos de semillas, por lo que se optó por utilizar la distribución 
binomial negativa en los correspondientes modelos (Zuur et al. 2009). En todos los GLMM se utilizaron los individuos anidados dentro de cada sitio como factores aleatorios y el tratamiento como factor fijo. En caso de $P$. mahaleb no se realizó el análisis para la cantidad de semillas, ya que cada fruto forma sólo una semilla (Amodeo 2014), por lo que la probabilidad de formación de frutos o semilla formadas es la misma. Por último, se evaluaron las diferencias entre tratamientos aplicando comparaciones múltiples por pares con la prueba de Tukey, utilizando el paquete multcomp en R (Hothorn et al. 2008).

Para evaluar la dependencia de polinizadores en diferentes regiones geográficas se compararon los resultados obtenidos en nuestro trabajo con estudios en los que se emplearon los mismos tratamientos de polinización para las mismas especies y en diferentes localizaciones. En el caso de $S$. junceum, comparamos nuestros resultados con un estudio realizado en Cytisus scoparius (L.) Link (=Spartium scoparium L.), especie filogenéticamente cercana a nuestra especie focal. Dichos datos fueron obtenidos por Parker (1997) en Estados Unidos, donde la especie es exótica. El número de repeticiones fue 200 para PC y 200 para C. Para G. monspessulana utilizamos datos de Parker y Haubensak (2002), también realizado en Estados Unidos, donde la especie es exótica; el número de repeticiones fue 100 en cada caso. Por otro lado, para $P$. mahaleb utilizamos datos de Jordano (1993) a partir de un trabajo llevado a cabo en España, donde la especie es nativa; el número de repeticiones fue 184 y 535 para PC y $C$, respectivamente. Por último, utilizamos datos de Mazzolari y colaboradores (2016) para $R$. rubiginosa en la Argentina, donde la especie es exótica y se comporta como invasora; el número de repeticiones fue 45 en PC y 45 en C. Se prestó particular atención a que los ensayos de polinización fueran hechos en base a la misma metodología para que las comparaciones fueran válidas. No se encontraron datos de otros estudios para la especie $R$. ulmifolius, por lo que no se realizó comparación con esa especie. Para el análisis, se calculó la dependencia de polinizadores como la diferencia de la proporción media de frutos formados bajo el tratamiento de polinización cruzada, menos la proporción media de frutos formados en los controles. Luego, para cada diferencia de medias se estimó un intervalo de confianza de $95 \%$ para poblaciones independientes de la siguiente forma:

$$
\left[\left(\overline{\times_{1}}-\overline{\times_{2}}\right) \pm \tau_{d f, 1-\alpha / 2} * \sqrt{\frac{\hat{S}_{1}^{2}}{n_{1}}+\frac{\hat{S}_{2}^{2}}{n_{2}}}\right]
$$

donde $\bar{X}_{1}$ y $\bar{X}_{2}$ corresponden a la proporción media de frutos formados bajo el tratamiento de polinización cruzada y controles respectivamente, $\tau$ es el estadístico de la diferencia de medias, df son los grados de libertad de la diferencia de medias, $\alpha$ el nivel de significación, $\hat{S}_{1}^{2}$ y $\hat{S}_{2}^{2}$ son la varianzas de la proporción de frutos formados bajo los diferentes tratamientos $\mathrm{y}$, por último, $\mathrm{n}_{1} \mathrm{y}$ $\mathrm{n}_{2}$ son el número de repeticiones para cada tratamiento. Los valores de la dependencia se calcularon con datos obtenidos de los estudios para cada especie y fueron comparados con valores de dependencia estimados de nuestros datos. Cuando los datos no estuvieron disponibles en el texto de los estudios, se obtuvieron de las figuras con la utilización del programa GetData (GetData Graph Digitizer 2.26.0.20). Se consideraron significativas las diferencias de las medias cuando no se solaparon los intervalos de confianza de nuestros valores con los de otros estudios.

Aunque la dependencia de polinizadores puede variar entre estudios, dicha variación puede estar relacionada con cambios en los valores de los controles (e.g., por variación climática) y no con el tratamiento de polinización cruzada. Es por ello que, adicionalmente, se realizó una comparación entre las medias de los tratamientos y controles de nuestros datos y los diferentes estudios. Aquí, la diferencia que se calculó fue la proporción media de frutos formados bajo el tratamiento de polinización cruzada para un estudio y especie determinado, menos la proporción media de frutos formados bajo el tratamiento de polinización cruzada de nuestro estudio para la misma especie. El mismo procedimiento se realizó para la diferencia de medias de los controles. Se calcularon los intervalos de confianza al 95\% mediante la ecuación antes explicada. En este caso, nuestros valores (tratamiento y control) se consideraron diferentes a los de otros estudios, cuando los intervalos de confianza no incluyeron el valor cero. Diferencias medias positivas y distintas de cero, indican que los valores de otros estudios son superiores a 
los nuestros (valores negativos indican lo opuesto).

\section{Resultados}

La probabilidad de formación de frutos fue variable para cada especie y para cada tratamiento. Del total de flores utilizadas en cada especie, en total se formaron $29 \%$ de frutos en S. junceum, $19 \%$ en G. monspessulana, $35 \%$ P. mahaleb, $60 \%$ en $R$. rubiginosa y $87 \%$ en R. ulmifolius (Tabla S1). Asimismo, el número de semillas formadas en cada tratamiento varió considerablemente entre las especies (Tabla S2).

En particular, para las leguminosas, las probabilidades de formación de frutos fueron diferentes según la especie, aunque ambas presentaron mayor probabilidad de formación de frutos en PC con respecto al control. En $S$. junceum, la probabilidad para el tratamiento PC resultó ser estadísticamente mayor con respecto a los tratamientos $\mathrm{A}$ y $\mathrm{C}$, y no se encontraron diferencias entre estos últimos (Figura 1; Tablas S1 y S2). En contraste, para G. monspessulana no se encontraron diferencias significativas entre tratamientos PC y A, aunque resultó ser mayor la probabilidad de formación de frutos bajo los tratamientos con respecto al control (Figura 1; Tablas S1 y S2). En el caso de la cantidad de semillas formadas, los resultados siguieron la misma tendencia que para los frutos (Figura 2a,b; Tabla S2).

En el caso de las rosáceas, P. mahaleb presentó diferencias significativas entre los tratamientos (PC-A) y entre PC y el control, y fue significativamente mayor la probabilidad de formación de frutos en PC en ambos casos (Figura 1; Tablas S1 y S2). Para las últimas dos especies, $R$. rubiginosa y $R$. ulmifolius, la probabilidad de que se forme una infrutescencia bajo los distintos tratamientos no presentó diferencias significativas entre ellos (PC-A) ni con respecto al control (PC-C, $\mathrm{A}-\mathrm{C}$ ) (Figura 1; Tablas S1 y S2). Al igual que para las infrutescencias, la cantidad de frutos bajo los distintos tratamientos no resultó ser estadísticamente diferente entre tratamientos para estas dos especies (Figura 2c,d; Tabla S2).

Nuestros valores de dependencia de polinizadores en las leguminosas difirieron

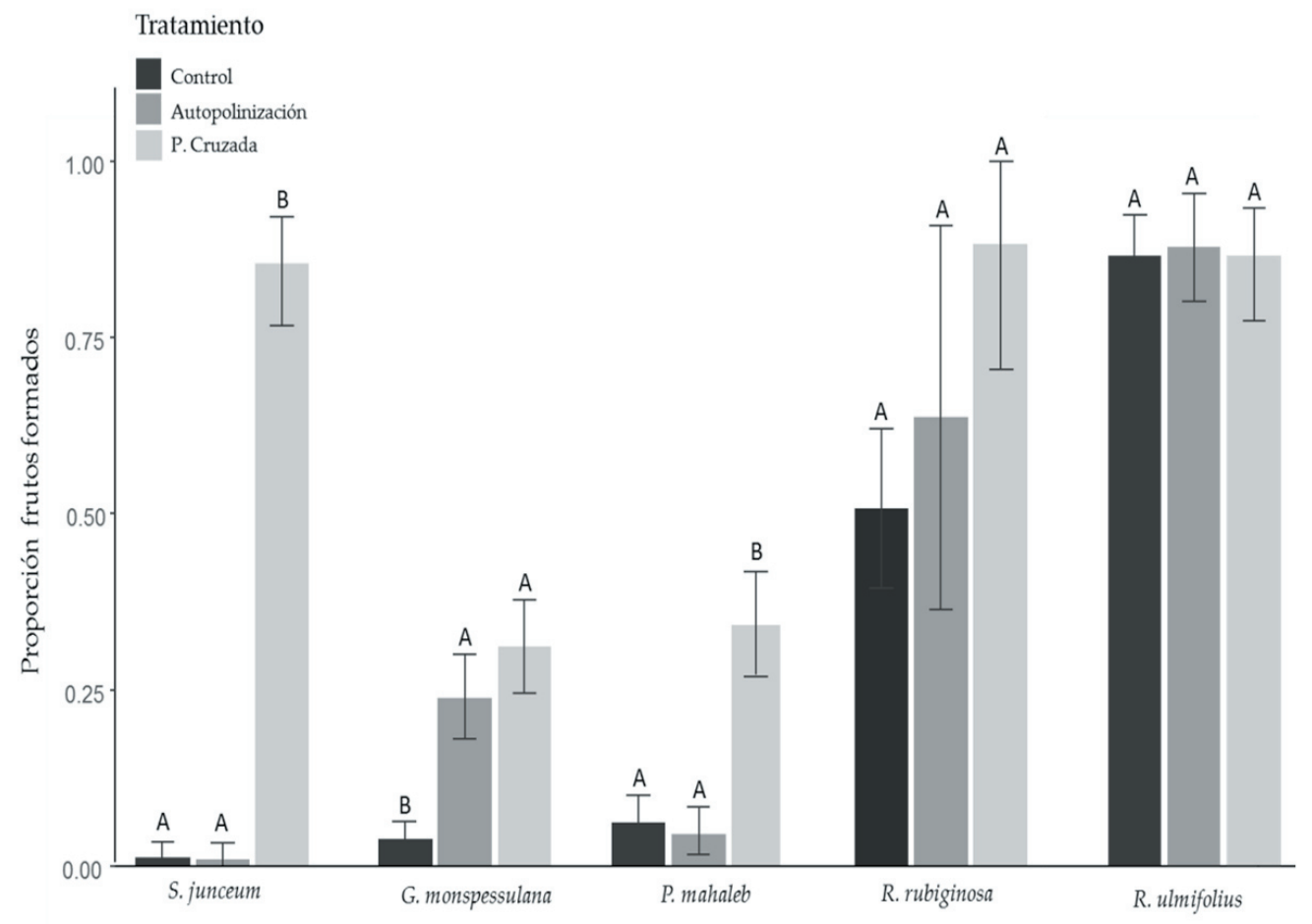

Figura 1. Proporción $( \pm E S)$ de frutos formados en cada especie y para cada tratamiento: control (negro), autopolinización (gris claro) y polinización cruzada (gris oscuro). Las comparaciones se hicieron entre los tratamientos y el control de cada especie por separado utilizando la prueba de contrastes de Tukey.

Figure 1. Proportion $( \pm$ SE) of developed fruits in each species for each treatment: control (black), self-pollination (dark gray) and cross-pollination (light grey). Comparisons were made between treatments and control of each species separately using the Tukey test. 

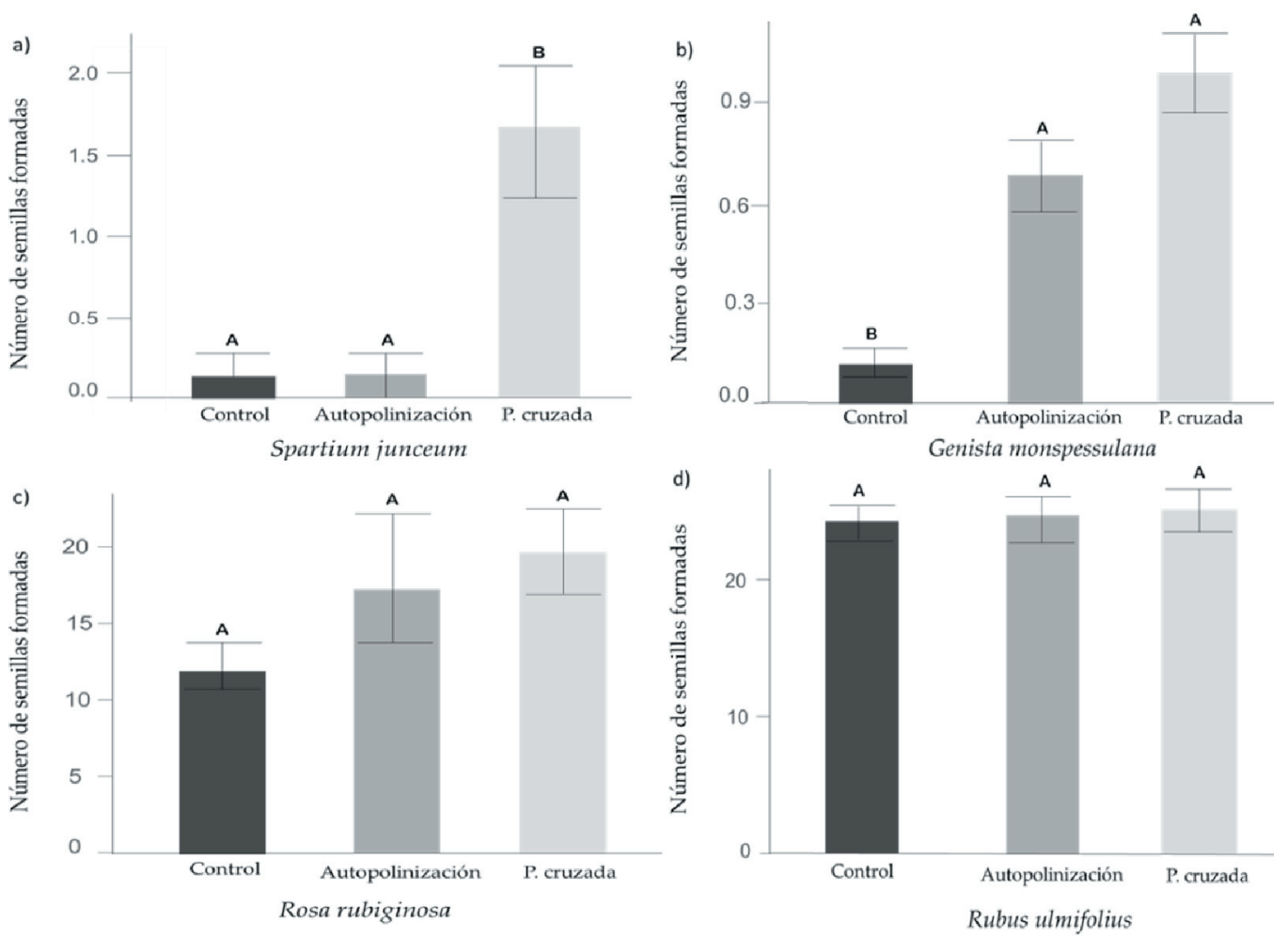

Figura 2. Número de semillas (media \pm ES) formadas en cada especie y para cada tratamiento: control, autopolinización y polinización cruzada. Las comparaciones se hicieron entre los tratamientos y el control de cada especie por separado utilizando la prueba de contrastes de Tukey.

Figure 2. Number of developed seeds (mean \pm SE) in each species and for each treatment: control, self-pollination and cross-pollination. The comparisons were made between treatments and control of each species separately using the Tukey test.
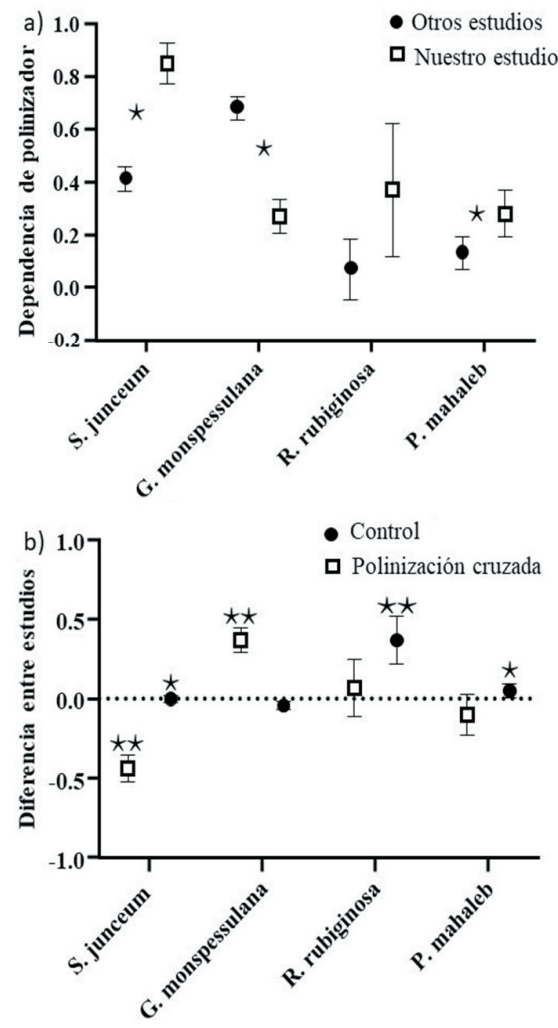

Figura 3. Comparación entre nuestro estudio y otros estudios en: (a) la dependencia de polinizadores, estimada como la diferencia de medias entre tratamientos de polinización cruzada y control (con nuestros datos y con datos de otros estudios obtenidos de bibliografía); (b) la diferencia de medias para polinización cruzada y control. Este valor es el resultado de la diferencia la proporción media de frutos formados para el tratamiento o el control de cada estudio y especie, menos la proporción media de frutos formados en nuestro estudio en la misma situación (tratamiento o control). Se calcularon intervalos de confianza del $95 \%$ para las diferencias de media (media $\pm \mathrm{IC}$ ). Las diferencias se consideraron altamente significativas $(\star \star)$ y significativas $(\star)$ cuando no se solaparon los intervalos de confianza en la misma especie (a) y los IC no incluyeron el valor 0 (b).

Figure 3. Comparison between our study and other studies in: (a) pollinator dependence, estimated as the difference in means between cross-pollination treatments and control (with our data and data from other studies obtained from bibliography); (b) the difference of means for cross-pollination and control. This value is the result of the difference between the average proportion of fruits formed for treatment or control in each study and species, minus the average proportion of fruits formed in our study in the same situation (treatment or control). 95\% confidence intervals were calculated for mean differences (mean $\pm \mathrm{CI})$. Differences were considered highly significant $(\star \star)$ and significant $(\star)$ when the confidence intervals in the same species did not overlap (a) and the $\mathrm{CI}$ did not include the value $0(\mathrm{~b})$. 
de otros estudios de manera opuesta. Para $S$. junceum, nuestros resultados fueron mayores a los de otros estudios, mientras que para G. monspessulana fueron menores (Figura 3a). En las rosáceas, nuestros resultados para $P$. mahaleb fueron mayores a los de otros estudios, mientras que en $R$. rubiginosa nuestros resultados no difirieron de los de otros trabajos (Figura 3a). Al comparar los controles de nuestros resultados con los de otros estudios encontramos la mayor diferencia para $R$. rubiginosa, diferencias menores para S. junceum y G. monspessulana, y ninguna diferencia para $P$. mahaleb (Figura $3 b$ ). Finalmente, se observaron diferencias entre los tratamientos de polinización cruzada para las leguminosas, mientras que no hubo diferencias para las rosáceas (Figura 3 b).

\section{Discusión}

La dependencia de polinizadores fue una característica variable entre las dos familias de plantas leñosas estudiadas en el PPET. Las especies que resultaron ser más dependientes del transporte de polen fueron las leguminosas. Tanto S. junceum como G. monspessulana tuvieron una mayor proporción de formación de frutos por polinización cruzada en contraste a los controles, lo que nos indica que la reproducción dentro del PPET está dada exclusivamente por la polinización biótica. En el caso de las rosáceas, $P$. mahaleb también mostró una dependencia del transporte de polen exógamo. Sin embargo, $R$. rubiginosa y $R$. ulmifolius mostraron un bajo grado de autoincompatibilidad. Ambas especies resultaron no depender de polinizadores y demostraron ser capaces de reproducirse de forma autógama, sin necesidad de vectores de polen exógamo. Cuando comparamos nuestros resultados con otros estudios, encontramos que si bien las especies se comportan de manera similar en cuanto a su dependencia, el grado en que dependen de los polinizadores puede variar según el sitio geográfico de estudio. Encontramos que la dependencia por polinizadores fue significativamente diferente para el caso de S. junceum y G. monspessulana con respecto a otros sitios. En el caso de $P$. mahaleb, la diferencia encontrada fue menor, y para $R$. rubiginosa no encontramos diferencias. Pudimos corroborar que las diferencias en las leguminosas estuvieron principalmente relacionadas con el tratamiento de polinización cruzada y no por los controles, a diferencia de las rosáceas, en las que los controles aportaron mayores diferencias.

\section{Dependencia de polinizadores en las especies estudiadas}

Las dos especies de leguminosas mostraron mayores porcentajes de formación de frutos en el tratamiento de PC, en contraste con el control, lo que indica una dependencia elevada de polinizadores por parte de ambas. En el caso de $S$. junceum, la formación de frutos bajo PC fue casi 100\% mayor que en A y C, lo cual indica que esta especie depende sólo de la reproducción cruzada para su fecundación. Estos resultados son coincidentes con dos estudios previos realizados por Parker (1997) y Parker y Haubensak (2002) en Cytisus scoparius. Estas autoras también encontraron que la probabilidad de formación de frutos es mayor en PC que en A en los dos estudios realizados, lo que muestra, al igual que nuestro estudio, la importancia de los polinizadores para la reproducción de dicha especie. En cuanto a G. monspessulana, la probabilidad de formación de frutos en PC fue mayor que en $C$, pero, a diferencia de la especie anterior, no se diferenció significativamente de A (Tablas S1 y S2). En particular, estos últimos resultados difieren de los encontrados por Parker y Hausenbak (2002), ya que ellas encontraron una diferencia significativa entre PC y A. Futuros estudios podrían investigar si la diferencia aquí detectada indica un cambio en la estrategia reproductiva de la especie. No obstante, la dependencia de polinizadores es igualmente elevada para esta especie, ya que necesita de un polinizador para abrir la flor (Parker and Haubensak 2002) y que ocurra polinización cruzada o autopolinización. Si la flor no es abierta por un polinizador, la probabilidad de que se forme un fruto es menor al 1\% (Parker and Haubensak 2002; este estudio).

El número de semillas formadas por tratamiento mantuvo la misma tendencia que para los frutos. La cantidad media de semillas para S. junceum fue mayor en PC que en A; por el contrario, en G. monspessulana no hubo diferencias entre tratamientos. Estos resultados coinciden con los hallados por Parker y Haubensak (2002), aunque es importante aclarar que ellas trabajaron con la cantidad de semillas sólo para frutos formados, y encontraron diferencias marginalmente significativas entre PC y A para el caso de C. scoparius, pero no para $G$. monspessulana. Esto resulta interesante ya que, según sus datos, G. monspessulana presentó menos depresión en la fecundidad de flores autopolinizadas que C. scoparius (Parker and Haubensak 2002), lo que podría relacionarse en nuestro estudio, al no encontrar diferencias 
para la formación de frutos en A con respecto a PC en G. monspessulana.

En el caso de Prunus mahaleb, resultó una especie muy dependiente de los polinizadores, ya que se obtuvo el máximo porcentaje de formación de frutos en PC con respecto al tratamiento A y al C. Los bajos valores de formación de frutos en A y C sugieren que su éxito en la reproducción depende principalmente de los polinizadores. Estos resultados coinciden con los obtenidos por Jordano (1993) en el sureste de España, donde la especie es nativa. En particular para los individuos hermafroditas, la especie resultó parcialmente autocompatible, aunque la mayor proporción de formación de frutos se dio en los individuos con polinización cruzada. El tratamiento control, con exclusión de polinizadores, también difirió significativamente de los otros tratamientos (Jordano 1993). La semejanza con nuestros resultados podría deberse a que los individuos que llegaron al PPET podrían no haber sufrido limitación por polinizadores, lo que podría determinar que el fenotipo no se vea modificado con respecto a su lugar de origen. Esto podría estar relacionado a que el elenco de polinizadores en ambas regiones sea similar en cuanto al servicio de polinización brindado (Jordano 1993; Amodeo 2014), aunque futuros estudios deberían corroborar esta hipótesis, ya que nuestro trabajo no evaluó el servicio de polinización ni los polinizadores en particular.

En el caso de Rosa rubiginosa, para la que la formación de frutos no varió con el tratamiento aplicado, los porcentajes de formación de frutos fueron altos para los tratamientos y el control obtuvo porcentajes también elevados. Por lo tanto, podemos concluir que esta especie es autógama y no dependiente (o de baja dependencia) de los polinizadores. Esto mismo reportaron Mazzolari y colaboradores (2016) en la reserva natural Villavicencio (Mendoza, Argentina), donde la especie actúa como invasora; en acuerdo a nuestros resultados tampoco encontraron diferencias entre ninguno de los tratamientos ni con respecto al control. En el caso de la formación de semillas, tampoco parece influir el tratamiento aplicado, aunque nuestro estudio solo se basó en cuantificar la cantidad de semillas formadas, Mazzolari y colaboradores (2016) determinaron que la viabilidad de las semillas no depende del tipo de polinización.

Por último, Rubus ulmifolius se comportó de manera muy similar a $R$. rubiginosa, aunque los porcentajes de formación de frutos fueron un poco mayores para los tratamientos y para el control. Del mismo modo que para los frutos, no se encontraron diferencias para la formación de semillas por fruto. Podemos concluir que la dependencia de los polinizadores en esta especie es baja y muy similar a la de $R$. rubiginosa, la cual posee una gran diversidad de estrategias reproductivas (Mazzolari et al. 2016). Aunque no se encontraron estudios sobre las estrategias reproductivas exclusivos para esta especie, y tampoco sobre la ecología de la polinización, a partir de bibliografía consultada sabemos que el género Rubus presenta una compleja variedad de sistemas reproductivos según las subespecies. En particular, R. ulmifolius se caracteriza por tener una reproducción exclusivamente sexual, con la formación de semillas a través de la doble fertilización (Šarhanová et al. 2012). No obstante, hay ejemplos de especies dentro de este género con diversas estrategias reproductivas, incluso asexuales como la apomixis (Monasterio-Huelin and Weber 1996).

\section{Distribución geográfica y relación filogenética}

A partir de nuestros resultados podemos sugerir que el contexto ecológico y el geográfico pueden determinar cambios sustanciales en las estrategias reproductivas de las plantas, como mencionan algunos autores (Parker and Haubensak 2002; Lozada-Gobilard et al. 2019). Por ejemplo, S. junceum y G. monspessulana presentaron diferencias significativas en la dependencia del servicio de polinización para nuestro sitio, en comparación con otros dos sitios de estudio, donde las especies también se comportaron como invasoras (Parker 1997; Parker and Haubensak 2002). En particular para G. monspessulana, un estudio reciente determinó que $88 \%$ del área hoy invadida por esta especie en el PPET es producto del avance en los últimos siete años, por lo que la reproducción de esta especie es exitosa dentro del área (Ramírez 2020). Podríamos pensar que la alta dependencia encontrada para las leguminosas se debió a que el servicio de polinización en nuestro sitio fue provisto de forma eficiente por polinizadores, lo que influyó en la selección del fenotipo encontrado. En futuros estudios sería interesante evaluar cuál es el servicio de polinización del lugar y cuáles los polinizadores efectivos, ya que podría estar determinando el avance de la especie dentro del PPET. Lamentablemente, no existen datos sobre el avance de S. junceum dentro del PPET, 
ya que sería de interés comparar el crecimiento demográfico de ambas especies con sus grados de la dependencia de polinizadores.

En el caso de P. mahaleb, la diferencia encontrada resultó significativa, aunque no tan marcada como para las leguminosas; es decir que la dependencia varió poco entre nuestro sitio y su área de distribución nativa (Jordano 1993). Esto podría relacionarse con que la diferencia encontrada se debió a los controles y no al tratamiento, lo que podría indicar que la misma se deba a una cuestión climática y no a un cambio en el sistema reproductivo. Por último, $R$. rubiginosa no presentó diferencias entre las medidas de dependencia con respecto al trabajo de Mazzolari y colaboradores (2016). Por lo tanto, podemos decir que estas dos especies se comportan de forma similar entre los sitios comparados. Los resultados aquí encontrados concuerdan con Parker y Haubensak (2002) en que el rol de los polinizadores en la limitación de las invasiones puede ser muy variable entre las comunidades invadidas y que esto dificulta la generalización sobre el comportamiento de especies invasoras. Por último, las especies con mayor relación filogenética como el caso de las leguminosas siguieron el mismo patrón de dependencia tal como esperábamos, aunque el resultado fue variable para las rosáceas. Podríamos pensar que esta variación este dada por un grado de parentesco más cercano entre $R$. rubiginosa y $R$. ulmifolius, lo que determinaría una dependencia similar entre ellas, en contraste con $P$. mahaleb.

\section{Conclusiones}

Los resultados encontrados para las leguminosas concuerdan con lo mencionado por algunos autores que consideran que la polinización es un proceso clave durante las invasiones (en particular para las plantas leñosas) y que las especies son capaces de atraer con éxito a polinizadores generalistas (Richardson et al. 2000; Brown et al. 2002; Memmott and Waser 2002; Morales and Aizen 2006; Olesen et al. 2008). Lo anterior podría relacionarse con la importancia de la reproducción cruzada, ya que las especies con mayor diversidad genética pueden tener mayores posibilidades de adaptarse a nuevas condiciones en un entorno diferente a su área nativa (Barrett and Schluter 2008; Barrett 2010). En contraste, las tres especies de rosáceas estudiadas variaron en sus grados de dependencia del transporte de polen. De esta manera, el proceso de invasión en las rosáceas no estaría limitado por los polinizadores y quizás sí por otra interacción mutualista como la dispersión de los frutos o la asociación con microbiota simbionte (Traveset and Richardson 2014).

Nuestros resultados sugieren que las especies autógamas no presentan mayores ventajas para la formación de semillas, en relación con las especies que dependen mucho de la polinización cruzada. Es decir que la autogamia podría no representar un beneficio para las especies leñosas durante el proceso de invasión, como propusieron diversos autores (Baker 1955; HarmonThreatt et al. 2009; Barrett 2010). Por lo tanto, el cambio de los sistemas reproductivos desde la reproducción cruzada hacia la autogamia, no sería una generalización para las plantas leñosas en los procesos de invasión como se ha propuesto (Lozada-Gobilard et al. 2019 y referencias en el mismo). No obstante, si la selección del fenotipo está dada por los polinizadores, podríamos pensar que las plantas que logran grados de autogamia elevados no deberían revertir esta situación al no depender de ellos para su reproducción, como es el caso de $R$. rubiginosa y R. ulmifolius. Resulta de particular interés entender cómo las especies muy dependientes de polinizadores logran establecer interacciones eficientes con polinizadores nativos o exóticos en su nueva área de distribución. Una pregunta interesante de abordar en futuros estudios es entender cómo se integran en las redes de interacciones planta-polinizador (Tylianakis 2008) y de qué forma afectan a las especies nativas de las Sierras australes Bonaerenses. Esto resalta la importancia del estudio de las interacciones facilitadoras, en particular la polinización, para entender la integración de especies exóticas en las comunidades.

Agradecimientos. Los autores agradecen a la administración del Parque Provincial Ernesto Tornquist por permitir el acceso para llevar a cabo este estudio. Este trabajo fue desarrollado como parte del PGI "Impacto de los disturbios sobre el funcionamiento ecosistémico de los pastizales naturales del Grupo Geológico Ventana" bajo la dirección de la Dra. A. E. de Villalobos, otorgado por la Universidad Nacional del Sur, Bahía Blanca; y con financiamiento del PICT 2017-3336. J. P. Torretta realizó valiosas correcciones que mejoraron la calidad del manuscrito. Parte de los instrumentos de laboratorio utilizados fueron donados por la organización "Neotropical Grassland Conservancy". 


\section{REFERENCIAS}

Allen, O. N., and E. K. Allen. 1981. Leguminosae: a source book of characteristics, uses, and nodulation. University of Wisconsin Press, Madison, Wisconsin, USA.

Amodeo, M. R. 2014. Dispersión del cerezo de Santa Lucía (Prunus mahaleb) en pastizales naturales del sur de la Provincia de Buenos Aires. Tesis Doctoral. Departamento de Biología, Bioquímica y Farmacia, Universidad Nacional del Sur, Bahía Blanca, Argentina. Pp. 217.

Amodeo, M. R., and S. M. Zalba. 2013. Wild cherries invading natural grasslands: unraveling colonization history from population structure and spatial patterns. Plant Ecology 214:1299-1307. https://doi.org/10.1007/s11258-013-0252-4.

Amodeo, M. R., and S. M. Zalba. 2017. Sex morphs and invasiveness of a fleshy-fruited tree in natural grasslands from Argentina. Botany 95:913-922. https://doi.org/10.1139/cjb-2017-0041.

Baker, H. G. 1955. Self-compatibility and establishment of long-distance dispersal. Evolution 9:337-349. http://doi.org/ 10.2307/2405656,

Barrett, R. D. H., and D. Schluter. 2008. Adaptation from standing genetic variation. Trends in Ecology and Evolution 23:38-44. https://doi.org/10.1016/j.tree.2007.09.008.

Barrett, S. C. H. 2010. Why reproductive systems matter for the invasion biology of plants. Pages 195-210 in D. M. Richardson (eds.). Fifty years of invasion ecology: the legacy of Charles Elton. Blackwell, Oxford, UK. https://doi.org/ 10.1002/9781444329988.ch15.

Barrett, S. C. H., and C. G. Eckert. 1990. Current issues in plant reproductive ecology. Israel Journal of Plant Sciences 39:1-2. https://doi.org/10.1080/0021213X.1990.10677130

Barrett, S. C. H., M. T. Morgan, and B. C. Husband. 1989. The dissolution of a complex genetic polymorphism: the evolution of self-fertilization in tristylous Eichhornia paniculata (Pontederiaceae). Evolution 43:1398-1416.https: //doi.org/10.1111/j.1558-5646.1989.tb0259.x.

Bilenca, D., and F. Miñarro. 2004. Identificación de áreas valiosas de pastizal en las pampas y campos de Argentina, Uruguay y sur de Brasil (AVPs). No. 504.73 (8) BIL. Fundación Vida Silvestre, Buenos Aires, Argentina.

Brown, B. J., R. J. Mitchell, and S. A. Graham. 2002. Competition for pollination between an invasive species (purple loosestrife) and a native congener. Ecology 83:2328-2336. https://doi.org/10.1890/0012-9658(2002)083[2328: CFPBAI]2.0.CO;2.

Burgos, J. J. 1971. El clima de la provincia de Buenos aires en relación con la vegetación. Instituto Nacional de Tecnología Agropecuaria, Argentina.

Clapham, A. R., T. G. Tutin, and D. M. Moore. 1987: Flora of the British Isles. Cambridge: Cambridge University Press.

Core Team, R. C. T. M. 2013. R: a language and environment for statistical computing. R Foundation for Statistical Computing, Vienna, Austria. URL: www.R-project.org.

Devaux, C., C. Lepers, and E. Porcher. 2014. Constraints imposed by pollinator behaviour on the ecology and evolution of plant mating systems. Journal of Evolutionary Biology 27:1413-1430. https://doi.org/10.1111/jeb.12380.

Eckert, C. G., S. Kalisz, M. A. Geber, R. Sargent, E. Elle, P.-O. Cheptou, C. Goodwillie, M. O. Johnston, J. K. Kelly, D. A. Moeller, E. Porcher, R. H. Ree, M. Vallejo-Marín, and A. A. Winn. 2010. Plant mating systems in a changing world. Trends in Ecology and Evolution 25:35-43. https://doi.org/10.1016/j.tree.2009.06.013.

Frangi, J. L., and O. J. Bottino. 1995. Comunidades vegetales de la Sierra de la Ventana, provincia de Buenos Aires, Argentina. Revista de la Facultad de Agronomía de La Plata 71:93-133.

Gervasi, D. D. L., and F. P. Schiestl. 2017. Real-time divergent evolution in plants driven by pollinators. Nature Communications 8:14691. https://doi.org/10.1038/ncomms14691.

Harmon-Threatt, A. N., J. H. Burns, L. A. Shemyakina, and T. M. Knight. 2009. Breeding system and pollination ecology of introduced plants compared to their native relatives. American Journal of Botany 96:1544-1550. https: //doi.org/10.3732/ajb.0800369.

Hothorn, T., F. Bretz, and P. Westfall. 2008. Simultaneous inference in general parametric models. Biometrical Journal 50:346-363. https://doi.org/10.1002/bimj.200810425.

Jordano, P. 1993. Pollination biology of Prunus mahaleb L.: deferred consequences of gender variation for fecundity and seed size. Biological Journal of the Linnean Society 50:65-84. https://doi.org/10.1111/j.1095-8312.1993.tb00919.x.

Jordano, P. 1994. Spatial and Temporal Variation in the Avian-Frugivore Assemblage of Prunus mahaleb: Patterns and Consequences. Oikos 71:479-491. https://doi.org/10.2307/3545836.

Kearns, C. A., and D. W. Inouye. 1993. Techniques for pollination biologists. University press of Colorado. Niwot, Colorado 80544, Estados Unidos.

Long, M. A., and C. M. Grassini. 1997. Actualización del conocimiento florístico del Parque Provincial Ernesto Tornquist. Informe final del Convenio de colaboración recíproca Ministerio de Asuntos Agrarios Provincia de Buenos Aires y Universidad Nacional del sur, Bahía Blanca, Argentina.

Lozada-Gobilard, S., M. Weigend, E. Fischer, S. B. Janssens, M. Ackermann, and S. Abrahamczyk. 2018. Breeding systems in Balsaminaceae in relation to pollen/ovule ratio, pollination syndromes, life history and climate zone. Plant Biology 21:157-166. https://doi.org/10.1111/plb.12905.

Mazzolari, A. C., and V. Comparatore. 2014. Invasión de Rubus ulmifolius (Rosacea) en la Reserva Integral Laguna de los Padres, Buenos Aires, Argentina: bases para el trazado de estrategias de manejo y recuperación del bosque nativo. BioScriba 7:19-29. 
Mazzolari, A. C., H. J. Marrero, and D. P. Vázquez. 2016. Potential contribution to the invasion process of different reproductive strategies of two invasive roses. Biol Invasions 18:1387-3547. https://doi.org/10.1007/s10530-016-1315y.

Memmott, J., and N. M. Waser. 2002. Integration of alien plants into a native flower-pollinator visitation web. Proceedings of the Royal Society of London. Series B: Biological Sciences 269:2395-99. https://doi.org/10.1098/rspb.2002.2174.

Monasterio-Huelin, E., and H. E. Weber. 1996. Taxonomy and nomenclature of Rubus ulmifolius and Rubus sanctus (Rosaceae). Edinburgh Journal of Botany 53:311-322. https://doi.org/10.1017/S0960428600003759.

Morales, C. L., and M. A. Aizen. 2006. Invasive mutualisms and the structure of plant-pollinator interactions in the temperate forests of north-west Patagonia, Argentina. Journal of Ecology 94:171-180. https://doi.org/10.1111/j.13652745.2005.01069.x.

Niet, T. V. D., and S. D. Johnson. 2012. Phylogenetic evidence for pollinator-driven diversification of angiosperms. Trends in Ecology and Evolution 27:353-361. https://doi.org/10.1016/j.tree.2012.02.002.

Olesen, J. M., J. Bascompte, H. Elberling, and P. Jordano. 2008. Temporal dynamics in a pollination network. Ecology 89:1573-1582. https://doi.org/10.1890/07-0451.1.

Parker, I. M. 1997. Pollinator limitation of Cytisus scoparius (Scotch broom), an invasive exotic shrub. Ecology 78:14571470. https://doi.org/10.2307/2266140.

Parker, I. M., and K. A. Haubensak. 2002. Comparative pollinator limitation of two non-native shrubs: do mutualisms influence invasions? Oecologia 130:250-258. https://doi.org/10.1007/s004420100799.

Parodi, L. R. 1978. Enciclopedia Argentina de Agricultura y Jardinería. Descripción de las plantas cultivadas. Tomo I. ACME SACI. Buenos Aires, Argentina.

Pauchard, A., R. García, E. Peña, C. González, L. Cavieres, and R. Bustamante. 2008. Positive feedbacks between plant invasions and fire regimes: Genista monspessulana (L.) K. Koch (Fabaceae) in central Chile. Biological Invasions 10: 547-553. https://doi.org/10.1007/s10530-007-9151-8.

Petanidou, T., A. S. Kallimanis, S. P. Sgardelis, A. D. Mazaris, J. D. Pantis, and N. M. Waser. 2014. Variable flowering phenology and pollinator use in a community suggest future phenological mismatch. Acta Oecologica 59:104-111. https://doi.org/10.1016/j.actao.2014.06.001.

Ramírez, F. J. 2020. Invasión de retamilla (Genista monspessulana) en las sierras australes bonaerenses: historia de su avance y estrategias de control. Tesis de grado. Departamento de Biología, Bioquímica y Farmacia, Universidad Nacional del Sur, Bahía Blanca. Argentina. Pp. 32.

Richardson, D. M., N. Allsopp, C. M. D'antonio, S. J. Milton, and M. Rejmánek. 2000. Plant invasions-the role of mutualisms. Biological Reviews 75:65-93. https://doi.org/10.1017/s0006323199005435.

Sanhueza, C. 2012. Ecología y manejo de leguminosas invasoras en la Sierra de la Ventana. Tesis Doctoral. Departamento de Biología, Bioquímica y Farmacia, Universidad Nacional del Sur, Bahía Blanca. Argentina. Pp. 166.

Šarhanová, P., R. J. Vašut, M. Dančák, P. Bureš, and B. Trávníček. 2012. New insights into the variability of reproduction modes in European populations of Rubus subgen. Rubus: how sexual are polyploid brambles? Sexual plant reproduction 25:319-335. https://doi.org/10.1007/s00497-012-0200-9.

Traveset, A., and D. M. Richardson. 2014. Mutualistic Interactions and Biological Invasion. Annual Review of Ecology, Evolution, and Systematics 45:89-113. https://doi.org/10.1146/annurev-ecolsys-120213-091857.

Tylianakis, J. M. 2008. Understanding the Web of Life: the Birds, the Bees and Sex with Aliens. PLoS Biology 6:e47. https://doi.org/10.1371/journal.pbio.0060047.

Ward, M., S. D. Johnson, and M. P. Zalucki. 2011. Modes of reproduction in three invasive milkweeds are consistent with Baker's Rule. Biological Invasions 14:1237-1250. https://doi.org/10.1007/s10530-011-0152-2.

Zalba, S. M. and C. B. Villamil. 2002. Woody plant invasion in relictual grasslands. Biological Invasions 4: 55-72. https: //doi.org/10.1023/A:1020532609792.

Zuur, A., E. N. Ieno, N. Walker, A. A. Saveliev, and G. M. Smith. 2009. Mixed Effects Models and Extensions in Ecology with R. Springer Science and Business Media. New York, USA. https://doi.org/10.1007/978-0-387-87458-6. 